\title{
Adherence to Antiretroviral Treatment for Prevention of Mother-to-Child Transmission of HIV in Eastern Ethiopia: A Cross-Sectional Study
}

This article was published in the following Dove Press journal: HIVIAIDS - Research and Palliative Care

\author{
Anwar Fedlu' \\ Birhanu Alie' \\ Ammas Siraj Mohammed (iD ${ }^{2}$ \\ Fuad Adem ${ }^{2}$ \\ Abduselam Hassen' \\ 'School of Public Health, College of \\ Health and Medical Sciences, Haramaya \\ University, Harar, Ethiopia; ${ }^{2}$ Department \\ of Clinical Pharmacy, School of Pharmacy, \\ College of Health and Medical Sciences, \\ Haramaya University, Harar, Ethiopia
}

Introduction: Even though PMTCT (prevention of mother-to-child transmission) is the most effective option to prevent vertical transmission of HIV, achieving optimal adherence has been a universal challenge to PMTCT, including Ethiopia.

Objective: To assess levels of adherence and associated factors among pregnant and lactating mothers on PMTCT at public health facilities in Harar town, Harari region, Eastern Ethiopia.

Methods: A facility-based cross-sectional study was conducted from April 01 to April 30, 2019, at the selected public health facilities of Harar town, Harari regional state, Eastern Ethiopia. Adherence was measured using a multi-method tool that includes pill counting and self-reporting method. Binary logistic regression analysis was used to determine factors associated with PMTCT adherence. The results were adjusted for possible confounding variables and P-value $<0.05$ or CI not including 1 was considered statistically significant.

Results: Of the 190 included patients, $83.2 \%$ of them had good adherence to PMTCT. Forgetfulness (79\%) and being busy (71\%) were among the main reasons for nonadherence to PMTCT. In multivariate logistic regression, participants who have received counseling on side effects were 3.4 times more likely to have good adherence [AOR $=3.4$, 95\% CI: 2.1-9.2]; those with previously known HIV status were 2.1 times more likely to have good adherence to PMTCT [AOR=2.1, 95\% CI: 1.6-7.1] and women who were knowledgeable on PMTCT were 5.2 times more likely to have good adherence [AOR=5.2, 95\% CI: 1.6-6.28].

Conclusion: In this study, the overall level of adherence to PMTCT care and support was $83.2 \%$. Counseling on side effects, knowledge about PMTCT, and pregnancy status during initiation of ART were the factors that determine adherence of pregnant and lactating women towards PMTCT service. Thus, it is recommended to raise awareness about PMTCT among all reproductive-age women by stressing the benefit of the early initiation of ART and by providing information on the possible side effect of the drugs.

Keywords: pregnant and lactating women, medication adherence, PMTCT, Harar, Ethiopia

\section{Introduction}

Prevention of mother-to-child transmission (PMTCT) is a comprehensive management approach largely aimed at preventing mother-to-child HIV transmission. ${ }^{1}$ With its "treat-all" recommendation, WHO removes all limitations on eligibility for ART among people living with HIV, including pregnant women and lactating women. Increasing evidence to support earlier ART initiation among all adults,
Correspondence: Ammas Siraj Mohammed Department of Clinical Pharmacy, School of Pharmacy, College of Health and Medical Sciences, Haramaya University, Harar, Ethiopia

Email ammassiraj2337@gmail.com 
emerging programme data on its success in practice, all supported a revised recommendation in 2015 that moved away from "options" for PMTCT and instead advocated that all pregnant and breastfeeding women living with HIV should initiate ART and remain on lifelong treatment, regardless of CD4 cell count or stage of disease (formerly called option $\left.\mathrm{B}^{+}\right)^{2}$

Ethiopia also adopted universal treatment in 2018 and advocated the importance of rapid initiation of ART in significantly reducing vertical transmission of HIV. A Fixed-dose combination of $\mathrm{TDF}+3 \mathrm{TC}+\mathrm{EFV}$ is the preferred dosage regimen for pregnant and breastfeeding women. ${ }^{3}$ Globally, new pediatrics HIV infections are 110 000 [78,000-150 000] in 2015. More than 90\% of these children are infected through Mother-to-Child Transmission (MTCT) and the overall transmission rate without ART is $20-45 \% .{ }^{4}$ African priority countries in the WHO Global Plan account for over $90 \%$ of all pregnant women living with HIV and new infections among children globally. ${ }^{5}$ In Ethiopia, an estimated 3800 [20006000] children were infected with HIV while 2900 [1700-4300] children were died due to HIV in $2016 .^{6}$

The global plan for the elimination of mother-to-child transmission of HIV scaled up by UNIADS among countries with the highest mother-to-child infection disease burden aimed to reduce MTCT of HIV to less than 5\% between 2009 and 2015. ${ }^{7}$ Despite this ambitious goal, about $86 \%$ of the estimated 160000 children newly infected with HIV in 2018 were in the WHO priority African region. ${ }^{8}$

Adherence to antiretroviral therapy (ART) has been shown to improve treatment outcomes among HIVinfected individuals. ${ }^{9}$ Though providing lifelong ART to pregnant and lactating women as the most effective option to prevent HIV transmission and resistance, achieving optimal adherence remained to be a universal challenge to PMTCT. ${ }^{10}$ Sub-optimal adherence to lifelong ART among pregnant and breastfeeding women has been among the causes of high MTCT rates in low- and middleincome countries. ${ }^{7,11}$

Ethiopia has implemented many efforts to reduce MTCT but it did not reach the global plan of an MTCT rate of $<5 \%$. Ethiopia has faced problems in adherence to ART, as a high percentage of pregnant mothers did not adhere to the full course of lifelong treatment for a variety of reasons which may lead to an increased risk of MTCT. $^{12}$
Previous studies in Ethiopia have reported an adherence level of below 95\%, which is insufficient to bring good health outcomes and reduction of viral loads by ART. $^{13,14}$ Forgetfulness, educational level, a side effect of the drug, undisclosed HIV status and fear of being stigmatized were among factors negatively affecting ART adherence. ${ }^{15-21}$ As universal treatment does not consider CD4 status and clinical stage, many women feel healthy to initiate lifelong ART, which is likely to effects their adherence. ${ }^{15}$ Another anticipated fear related to option B + was that; it recommends initiation of lifelong ART without considering gestational age, as early as their diagnosis, which may increase the probability of non-adherence due to long-time exposure to treatment. ${ }^{22}$

Only a few studies assessed ART adherence during pregnancy in Ethiopia and such studies did not explore ART adherence among lactating women. Furthermore, none of the previous studies addressed PMTCT adherence issues among HIV-positive pregnant and lactating women in Harari regional state. Therefore, the current study aimed at assessing the level of PMTCT adherence and associated factors among pregnant and lactating women in Harar town, Harari regional state, Eastern Ethiopia.

\section{Materials and Methods \\ Study Area, Design, and Setting}

This is a facility-based cross-sectional study conducted on 190 pregnant and lactating mothers on PMTCT follow-up at the selected public health facilities of Harar town. Harar is an ancient town located at $526 \mathrm{~km}$ east of Addis Ababa, the capital of the nation. The region has a total population of 232,000. Concerning the health care delivery system and disease pattern, it has a higher degree of health service coverage. According to the current regional health bureau profile, the Harari National Regional State (HNRS) has four governmental, two private, and one non-government (Fistula) hospitals; a total of seven hospitals, eight health centers, twenty-nine private clinics, twenty-six health posts and one regional laboratory serving the peoples of the state and surrounding woreda (unpublished data from Harari regional health bureau, 2019). The study was conducted from April 01 to April 30, 2019 GC.

\section{Population}

All pregnant women and lactating mothers on PMTCT follow up at the selected public health facilities of Harar town were source population whereas pregnant women 
and lactating mothers who were visiting the ART clinic in each health facility during the data collection period were considered as the study population.

\section{Eligibility Criteria}

HIV-positive pregnant and lactating mothers, who were attending the PMTCT programme at the time of the study and who had been on ART for at least four weeks were included in the study. Pregnant Women and lactating mothers, who were critically ill and who refused to participate were excluded.

\section{Sampling and Recruitment Strategies}

The desired sample size was calculated using a single proportion formula, taking into account a 95\% confidence interval, $5 \%$ margin of error, and $87.1 \%$ level of ART adherence, ${ }^{14}$ and $10 \%$ was considered for possible non-response rate to come up with a final sample size of 190. A total of seven health facilities were purposively selected by considering the large number of HIV-positive pregnant and lactating women diagnosed and enrolled in the PMTCT program at these facilities. A systemic random sampling technique was utilized to recruit the study subject $(\mathrm{k}=2)$ until the required sample size was achieved.

\section{Data Collection and Management}

The data collection was done by using an intervieweradministered questionnaire on PMTCT treatment adherence and its associated factors among pregnant and lactating mothers. The data collection was performed by three well-trained nurses working at the ART clinic and necessary training was provided to data collectors on contents of the questionnaire, data collection methods and ethical concerns by the principal investigator. Data consistency was checked by the principal investigator daily. The questionnaire was created by modifying items in the previous study on PMTCT treatment adherence and its predictors. ${ }^{17}$ The tool was pretested on 20 voluntary HIV-positive pregnant lactating women who were not included in the final survey and necessary modifications were instituted to the final data collection tool. The tool was checked for reliability with a Cronbach alpha value of 0.792 . The content of the tool was reviewed by a team of experts including internal medicine specialists and nurses working in the ART clinics. Adherence was measured using a multi-method tool to measure ART adherence in resource-constrained settings that includes pill counting and self-reporting method. ${ }^{23}$ If measurement using the two methods results in discordant results; adherence was determined using the selfreporting method, due to its low recall bias and being the recommended method for developing countries. Knowledge was assessed using six questions that could rate the knowledge level of study participants towards PMTCT. The knowledge considered good if the score of the knowledge question is 3 or above and poor if the score is less than 3. Similarly, four queries were used to determine the attitude of study participants. Participants who have correctly answered two or more questions were considered to have a good attitude while those who scored less than average were considered as having a poor attitude toward PMTCT. Male partner involvement in the PMTCT program was measured using 10 questions that focus on the male partner's support of his wife, with a minimum score of 0 and a maximum of 10 . Involvement was considered "good" for those who scored $\geq 7$, "moderate" for those who scored 4-6, and "low" for those who scored $\leq 3$ to the questions on male partner support. ${ }^{14}$

\section{Variables}

Dependent Variables

Level of Adherence to PMTCT

\section{Independent Variables}

Sociodemographic characteristics: age, income, educational status, marital status, occupation, place of residence. Health service delivery factors: distance of health facility, type of health facility, adherence counseling. Treatment and drug-related factors: side effects of ARV drugs, CD4 status and WHO clinical stages, challenges faced in sameday diagnosis and initiating art. Patient-related factors: knowledge, attitude, disclosure status, forgetfulness, and male partner involvement.

\section{Operational Definition Good Adherence}

Missing not more than one pill in a month prior to the interview and responded correctly to two or more of the four self-report measurement questions.

\section{Poor Adherence}

Missing more than one pill within a month and responding correctly to less than two of the four self-report measurement questions. 


\section{Statistical Processing and Analysis}

The collected data were checked for accuracy and completeness and entered and analyzed using statistical package for social science (IBM-SPSS) software version 21.0. Descriptive statistics such as proportions, means and medians were performed for categorical and continuous variables. Bivariate logistic regression was performed and variables having a significant association with the level of adherence at a 0.25 significance level were entered into multivariate logistic regression analyses to determine predictors of adherence to PMTCT. An odd ratio at $95 \% \mathrm{CI}$ and a p-value $<0.05$ was considered significant.

\section{Ethical Approval}

The study protocol and verbal consent were approved by the Institutional Health Research Ethics Review Committee of the College of Health and Medical Sciences, Haramaya University. An official letter was obtained from the School of public health (protocol. No. C/Ac/R/D//36/2019) and given to the DRH administration to secure permission. All participants provided informed verbal consent to take part in the study and the collected data were kept anonymous. This study was conducted following the Declaration of Helsinki.

\section{Result}

\section{Sociodemographic Characteristics of} Respondent

A total of 190 HIV-positive pregnant and lactating mothers were approached with a response rate of $100 \%$. Of the study participants, around a third (34.7\%) of them were within 25-29 age groups and the majority $74.7 \%$ (142) of respondents were urban residents. Concerning the educational status, more than half $(55.7 \%)$ of the respondents have attended secondary education and above. Almost all (94.7\%) participants were married and seventy-two $(37.9 \%)$ of the respondents were housewives. The majority (73.7\%) of women have attended the hospital for the service and a hundred twenty $(63.16 \%)$ of the respondent had a monthly income of greater than 50 USD. Among participants, more than two-thirds of them have experienced drugs' side effects, with nausea 103 (72.53\%) and vomiting 78 (54.92\%) were the most reported side effects. The difficulty of the early decision was the most $128(67.4 \%)$ depicted challenges faced by respondents in same-day diagnosis and initiating ART $128(67.4 \%)$ and the majority of them have disclosed their HIV status (Table 1).

\section{Knowledge, Attitude, and Having Male Partner Support of Study Participants}

Among study participants, 168 (88.4\%) of study participants had good knowledge of PMTCT; the majority 139 (73.1\%) of study participants had a positive attitude towards PMTCT. Of $180 \mathrm{HIV}^{+}$women claimed to live with their husbands, only $72(40 \%)$ of them reported having good male partner support (Table 2).

\section{Level of Adherence to PMTCT Drugs}

Using a multi-method tool to measure ART adherence in resource-constrained settings that includes pill counting and self-reporting method, the majority (83.2\%) of study participants revealed to have good adherence while the remaining $32(16.8 \%)$ were considered to have poor adherence to PMTCT.

\section{Reasons for Non-Adherence to PMTCT}

In this study, forgetfulness (79\%) and being busy (71\%) were the main reasons for non-adherence while depression, lack of support from a male partner, fear of side effects and social stigma, difficulties in the same-day decision of initiating lifelong ART were also the reasons that claimed by study participants (Figure 1).

\section{Level of Adherence to PMTCT and Its Predictors}

In multivariate logistic regression, previously known HIV status was positively associated with the level of adherence to PMTCT; participants who had previously known HIV status were 2.1 times more likely to have good adherence to PMTCT than those who were put on ART after pregnancy $[\mathrm{AOR}=2.1,95 \% \mathrm{CI}: 1.6-7.1]$. Women who were knowledgeable on PMTCT were 5.2 times more likely to have good adherence than those who had poor knowledge of PMTCT [AOR=5.2, 95\% CI: 1.6-6.28]. Finally, participants who have received counseling on side effects were 3.4 times more likely to have good adherence than their counterparts who have never received counseling on side effects [AOR=3.4, 95\% CI: 2.1-9.2] (Table 3). 
Table I Sociodemographic Characteristics of HIV+ Pregnant and Lactating Mothers in Selected Government Health Facilities of Harari Region, Harar, Eastern Ethiopia, 2019

\begin{tabular}{|c|c|c|c|}
\hline Variables & Characteristics & $\mathbf{n}$ & $\%$ \\
\hline \multirow[t]{4}{*}{ Age } & $<25$ & 45 & 23.7 \\
\hline & $25-29$ & 66 & 34.7 \\
\hline & $30-34$ & 46 & 24.2 \\
\hline & $>35$ & 33 & 17.4 \\
\hline \multirow[t]{2}{*}{ Residence } & Urban & 142 & 74.7 \\
\hline & Rural & 48 & 25.3 \\
\hline \multirow[t]{2}{*}{ Marital status } & Married & 180 & 94.7 \\
\hline & Single & 10 & 5.3 \\
\hline \multirow[t]{4}{*}{ Educational status } & No formal education & 37 & 19.5 \\
\hline & Primary school & 47 & 24.7 \\
\hline & Secondary school & 51 & 26.8 \\
\hline & Higher education & 55 & 28.9 \\
\hline \multirow[t]{6}{*}{ Occupational states of participants } & House wife & 72 & 37.9 \\
\hline & Private employee & 43 & 22.6 \\
\hline & Merchant & 37 & 19.5 \\
\hline & Government & 36 & 18.9 \\
\hline & employee & & \\
\hline & Student & 2 & I.I \\
\hline \multirow[t]{2}{*}{ Study participants current condition } & Pregnant & 130 & 57.4 \\
\hline & Lactating & 60 & 42.6 \\
\hline \multirow[t]{2}{*}{ Monthly income in USD } & $\leq 50$ & 70 & 36.4 \\
\hline & $>50$ & 120 & 63.16 \\
\hline \multirow[t]{2}{*}{ Health institution of follow up } & Hospital & 140 & 73.7 \\
\hline & Health center & 50 & 26.3 \\
\hline \multirow[t]{3}{*}{$\mathrm{CD}_{4}$ count at initiation of $\mathrm{ART}$} & $<350$ & 78 & 41.05 \\
\hline & $\geq 350$ & 74 & 38.95 \\
\hline & $\begin{array}{l}\text { Not measured at } \\
\text { initiation }\end{array}$ & 38 & 20 \\
\hline \multirow[t]{3}{*}{ WHO clinical category at admission } & Stage one & 102 & 53.7 \\
\hline & Stage two & 74 & 38.9 \\
\hline & Stage three & 14 & 7.4 \\
\hline \multirow{2}{*}{$\begin{array}{l}\text { Have experienced any side effects of } \\
\text { the drug }\end{array}$} & Yes & 142 & 74.7 \\
\hline & No & 48 & 25.3 \\
\hline \multirow[t]{4}{*}{ The experienced side effects } & Nausea & 103 & 72.53 \\
\hline & Vomiting & 78 & 54.92 \\
\hline & Dizziness & 26 & 18.30 \\
\hline & Skin rash & 54 & 38 \\
\hline \multirow[t]{4}{*}{$\begin{array}{l}\text { Challenges faced in same-day } \\
\text { diagnosis and initiating ART }\end{array}$} & $\begin{array}{l}\text { Difficulty of early } \\
\text { decision }\end{array}$ & 128 & 67.4 \\
\hline & $\begin{array}{l}\text { Fear of the side } \\
\text { effect of drug }\end{array}$ & 21 & II.I \\
\hline & Fear of stigma & 77 & 40.5 \\
\hline & $\begin{array}{l}\text { Difficulty of discloser } \\
\text { to partner }\end{array}$ & 83 & 43.7 \\
\hline \multirow[t]{2}{*}{ Disclosed HIV status } & Yes & 166 & 87.37 \\
\hline & No & 24 & 12.3 \\
\hline
\end{tabular}

Table 2 Knowledge and Attitude of Study Participants Towards PMTCT, Harari Region, Harar, Eastern Ethiopia 2019

\begin{tabular}{|c|c|}
\hline Variables & Frequency (\%) \\
\hline \multicolumn{2}{|l|}{ Knowledge } \\
\hline Good & $168(88.4 \%)$ \\
\hline Poor & $22(11.6 \%)$ \\
\hline \multicolumn{2}{|l|}{ Attitude } \\
\hline Positive & $139(73.1 \%)$ \\
\hline Negative & $51(16.9 \%)$ \\
\hline \multicolumn{2}{|l|}{ Male partner involvement in PMTCT } \\
\hline Good & $72(40 \%)$ \\
\hline Moderate & $4 I(22.7 \%)$ \\
\hline Poor & $67(37.3 \%)$ \\
\hline
\end{tabular}

\section{Discussion}

This study has tried to assess the level of adherence to PMTCT and associated factors among pregnant and lactating women in the Harari region. In this study, the overall level of adherence to PMTCT care and support was $83.2 \%$. This finding is lower compared to a study conducted in Bwailan Hospital, Malawi (91\%). ${ }^{24}$ This discrepancy could be explained by the use of a more stringent method to assess the adherence in the current study; a multimethod tool that includes pill counting and self-reporting method was utilized. However, it was comparable with the study report from Zambia (82.5\%) and Kisumu in Kenya $(82 \%) .{ }^{25,26}$ This might be due to the adoption of universal treatment ART strategy by these nations.

Similarly, it is lower than previous studies on the level of adherence to option B+ PMTCT drugs among HIVpositive pregnant and breastfeeding women in Ethiopia: wollo zone, northeast Ethiopia 87.9\%, Tigray Region of north Ethiopia (87.1\%), and Yirgalem Hospital, south Ethiopia (88.7\%). ${ }^{14,17,27}$ This discrepancy could be due to sociodemographic differences in the study population of the study area from these regions of Ethiopia.

However, this level of adherence is higher than in studies carried out in Sobi Specialist Hospital, Nigeria (73.3\%), and in Tikur Anbessa Specialized Hospital, Ethiopia $(72.9 \%){ }^{13,28}$ The reason for low adherence in other studies may have been that they did not include pregnant and breastfeeding mothers. Pregnant and breastfeeding mothers face many challenges in addition to those faced by the general population, such as nausea, vomiting, and caring for their family and children. 


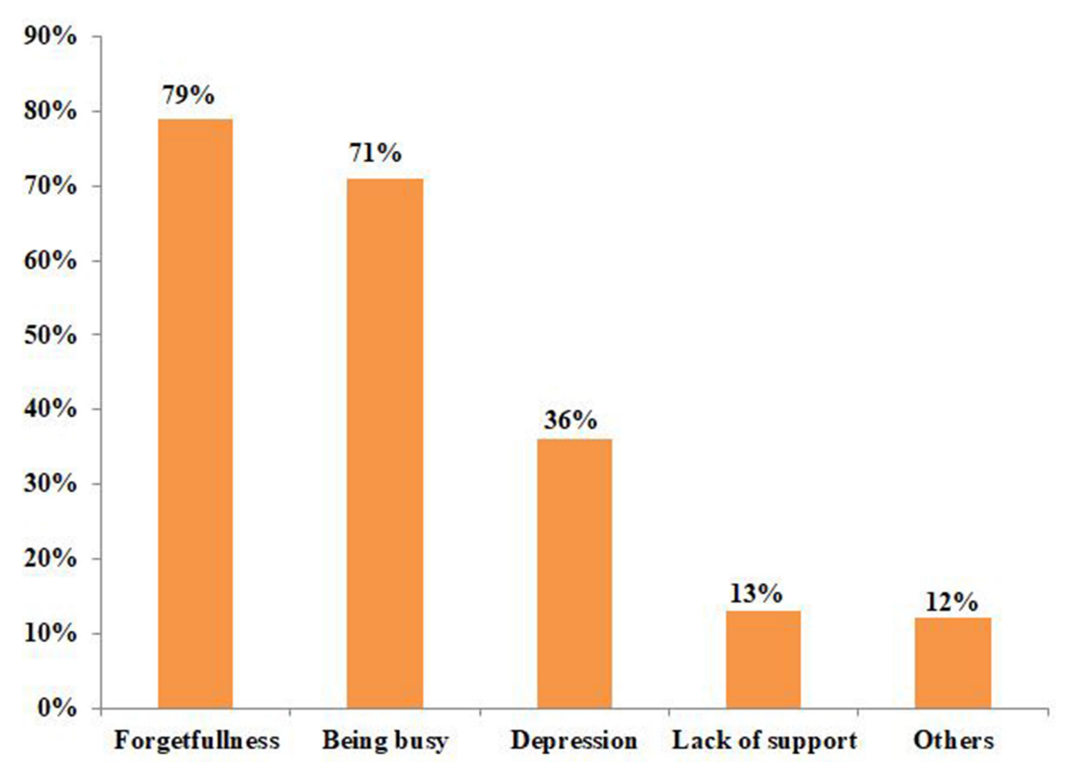

Figure I Reasons for non-adherence to PMTCT drugs, Harari region, Harar, Eastern Ethiopia, 2019. Others: include, fear of side effect, social stigma and difficulty in the same-day decision to initiate lifelong ART. The percentages add up to more than $100 \%$. PMTCT, prevention of mother-to-child transmission.

However, it is lower than other studies on the level of adherence to option B+ PMTCT drugs among HIVpositive pregnant and breastfeeding women in Ethiopia: wallo zone, northeast Ethiopia $87.9 \%$, Tigray Region of north Ethiopia (87.1\%), and Yirgalem Hospital, south Ethiopia (88.7\%). ${ }^{14,17,28}$ This discrepancy could be due to sociodemographic differences in the study population of the study area from these regions of Ethiopia.

The study revealed that women who were knowledgeable on PMTCT were 5.2 times more likely to have good adherence than those who had poor knowledge of PMTCT. This could be justified by Poor or inappropriate knowledge of pregnant and lactating women towards PMTCT care and Support causes' hesitation on the utilization of PMTCT and associated non-adherence. This indicates the need for exerting more effort to teach mothers about MTCT and PMTCT of HIV to improving the mothers' adherence to the care. Participants who had previously known HIV status were 2.1 times more likely to have good adherence to PMTCT than those who put on ART after pregnancy. This could be due to prolonged exposure to ART services and counseling of those with previously known HIV statuses. This shows that provider-initiated HIV counseling and testing (PICT) services should be provided for all women of childbearing age who are visiting the health institution.

The main reason for non-adherence in this study was forgetfulness (78\%). This is in agreement with the studies carried out in Nnewi, Nigeria (63.8\%), Lagos, Nigeria (57.6\%), and Addis Ababa health centers $(20.8 \%){ }^{29-31}$ In this study, participants who have received counseling on side effects were 3.4 times more likely to have good adherence than their counterparts who have never received counseling on side effects. This is corroborated by a study conducted in north Ethiopia which revealed proper counseling on the side effects of ARV drugs as a significant predictor of better adherence to PMTCT. ${ }^{14}$

\section{Limitations and Strength of the Study}

The study assessed the level of adherence to PMTCT and associated factors among pregnant and lactating women in the Harari region where such studies are limited. Therefore, the result of this study could be used as a reference for other studies on this topic and the results could serve as a baseline for program implementation. As a limitation, this study tried to assess adherence to PMTCT and associated factor using a self-reported survey, which may be subjected to 
Table 3 Level of Adherence to PMTCT and Its Predictors Among HIV-Positive Women in Selected Health Facilities in Harari Region, Harar, Eastern Ethiopia 2019

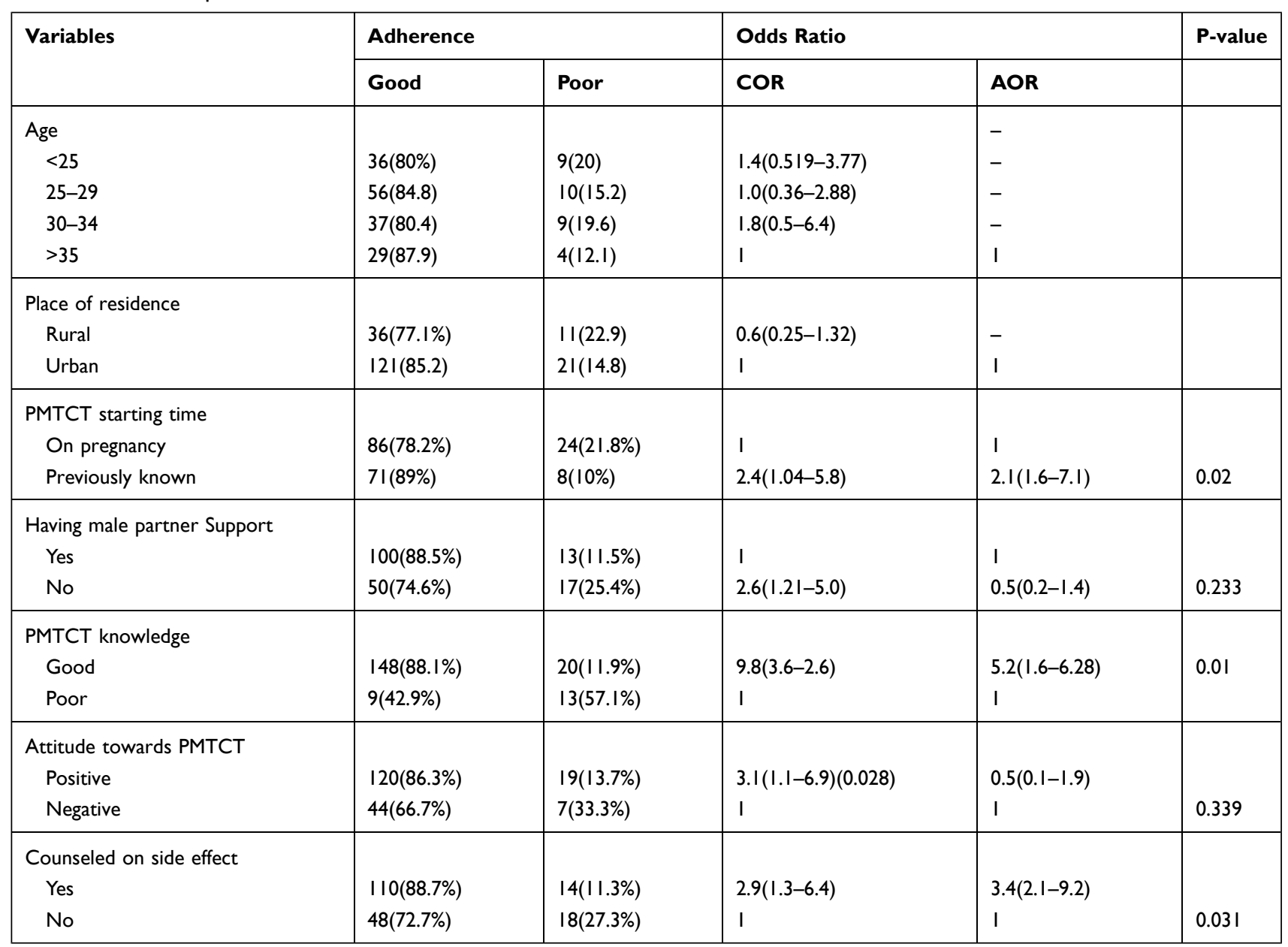

recall and social desirability biases. Subjects from several health facilities in the region have included addressing the generalizability limitation of the study.

\section{Conclusion and Recommendation}

In this study, the overall level of adherence to PMTCT care and support was $83.2 \%$. Counseling on side effects, knowledge about PMTCT, and pregnancy status during initiation of ART were the factors that determine adherence of pregnant and lactating women to PMTCT. Thus, it is recommended to create awareness about PMTCT among pregnant and lactating by stressing the benefit of the early start of ART and the possible side effect of the drugs.

\section{Abbreviations}

AIDS, Acquire Immune Deficiency Syndrome; ANC, Antenatal Care; ART, Antiretroviral Therapy; ARV, Antiretroviral; HIV, Human Immune Virus; MTCT, Motherto-Child Transmission; PMTCT, Prevention of Mother-to-
Child Transmission; SPSS, Statistical Package for Social Science; UN, United Nation; UNAIDS, Joint United Nations Programme on HIV/AIDS.

\section{Data Sharing Statement}

The data that support this study are available from the corresponding author on reasonable request.

\section{Consent for Publication}

Not applicable.

\section{Acknowledgments}

The authors are thankful to all individuals who were involved in the research.

\section{Author Contributions}

All authors made substantial contributions to conception and design, acquisition of data, or analysis and interpretation of data; took part in drafting the article or revising it critically 
for important intellectual content; gave final approval of the version to be published; and agree to be accountable for all aspects of the work.

\section{Funding}

This study received no support from a funding agency.

\section{Disclosure}

The authors declare no conflict of interest.

\section{References}

1. WHO PMTCT. Strategic Vision 2010-2015, preventing mother-tochild transmission of HIV to reach the UNGASS and Millennium Development Goals. 2010.

2. WHO consolidated guidelines on the use of antiretroviral drugs for treating and preventing HIV infection recommendations for a public health approach second edition. 2016

3. Ethiopian National consolidated guidelines for comprehensive HIV Prevention, care and treatment. 2018

4. Prevention of mother-to-child transmission (PMTCT) of HIV, MSF International AIDS Working Group Revised August. 2017

5. Joint United Nations Programme on HIV/AIDS (UNAIDS) (May 2015-April 2016). UNAIDS's support for the New Partnership for Africa's Development (NEPAD). Available from: http://www.unaids. org/sites/default/files/media_asset/GlobalPlan2016_en.pdf

6. WHO countries profile of HIV, Ethiopia; 2016

7. Joint United Nations Program on HIV/AIDS (UNAIDS). Prevention gap report. 2016.

8. WHO prevention of mother to child transmission of HIV, global health update. 2019

9. Omonaiye O, Kusljic S, Nicholson P, et al. Medication adherence in pregnant women with human immunodeficiency virus receiving antiretroviral therapy in sub-Saharan Africa: a systematic review. $B M C$ Public Health. 2018;18:805. doi:10.1186/s12889-018-5651-y

10. Kim MH, Zhou A, Mazenga A, et al. Why did i stop? Barriers and facilitators to uptake and adherence to ART in option B + HIV care in Lilongwe, Malawi. PLoS One. 2016;11(2):e0149527. doi:10.1371/ journal.pone. 0149527

11. Joint United Nations Program on HIV/AIDS (UNAIDS). A guide on indicators for monitoring and reporting on the health sector response to HIV/AIDS. 2017

12. Federal Ministry of Health. National Comprehensive PMTCT/ MNCH/RH Training Package Guideline Reference Manual. Addis Ababa: FMOH; 2013.

13. Demessie R, Mekonnen A, Amogne W, Shibeshi W. Knowledge and adherence to antiretroviral therapy among adult people living with HIV/AIDS at Tikur Anbessa Specialized Hospital, Ethiopia. Int J Basic Clin Pharmacol. 2014;3:320-330.

14. Ebuy H, Yebyo H, Alemayehu M. International journal of infectious diseases level of adherence and predictors of adherence to the option B + PMTCT program in Tigray, northern Ethiopia. Int Soc Infect Dis. 2015;33:123-129. doi:10.1016/j.ijid.2014.12.026

15. Karia JM, Factoring affecting access and utilization of PMTCT services in Nairobi province, Kenya. 44th International Course in Health Development (ICHD) September 24th 2007 September 12th; 2008; Netherland, Amsterdam.
16. Kirsten I, Sewangi J, Kunz A, et al. Adherence to combination prophylaxis for prevention of mother-to-child-transmission of HIV in Tanzania. PLoS One. 2011;6:e21020. doi:10.1371/journal. pone. 0021020

17. Tsegaye D, Deribe L, Wodajo S, et al. Levels of adherence and factors associated with adherence to option $\mathrm{B}+$ prevention of mother-to-child transmission among pregnant and lactating mothers in selected government health facilities of South Wollo Zone, Amhara Region, northeast Ethiopia, 2016. Epidemiol Health. 2016;38:e2016043. doi:10.4178/epih.e2016043

18. Juhar A. Level of adherence and associated factors to option $\mathrm{B}+$ PMTCT among HIV positive pregnant women in Hadiya Zone, Southern Ethiopia. Glob J Health Sci. 2017.

19. Mirkuzie AH, Hinderaker SG, Sisay MM, Moland MM, Morkve O. Current status of medication adherence and infant follow up in the prevention of mother to child HIV transmission programme in Addis Ababa. J Int AIDS Soc. 2011;14:50. doi:10.1186/1758-2652-14-50

20. Tarekegn M, Baru A, Seme A, et al. Levels of option B+ ART drugs adherence and associated factors among pregnant women following ART services at public health facilities of East Shawa Zone, Oromia, Ethiopia. Sex Reprod Healthc. 2019;22:100459. doi:10.1016/j. srhc.2019.100459

21. Balcha TT, Lecerof SS, Jeppsson AR, et al. Strategic challenges of PMTCT program implementation in Ethiopia. $J$ Int Assoc Physicians AIDS Care. 2011;10:187-192. doi:10.1177/1545109710369935

22. Kirsten I, Sewangi J, Kunz A, et al. Adherence to combination prophylaxis for prevention of mother-to-child-transmission of HIV in Tanzania. PLoS One. 2011;6:e21020. doi:10.1371/journal.pone.0021020

23. Steel G, Nwokike J, Joshi MP Development of a multi-method tool to measure ART adherence in resource-constrained settings: the South Africa experience; 2007. Available from: http:/apps.who.int/medicine docs/documents/s21890en/s21890en.pdf.

24. Ng'ambi WF, Tweya H, Speight C, Man-Bourdon C, Hosseinpour M, Phiri S. Determinants of antiretroviral treatment adherence among women accessing PMTCT "Option B+": a retrospective study at Bwaila Hospital, Malawi. IAS Conference on HIV Pathogenesis, treatment and prevention, 30 June-03 July2013-Kuala Lumpur, Malaysia, MOPE129-Poster Exhibition; 2013

25. Okawa $\mathrm{S}$, Chirwa $\mathrm{M}$, Ishikawa $\mathrm{N}$, et al. Longitudinal adherence to antiretroviral drugs for preventing mother-to-child transmission of HIV in Zambia. BMC Pregnancy Childbirth. 2015;15:258. doi:10.1186/s12884-015-0697-7

26. Thomas TK, Masaba R, Borkowf CB, et al. Triple-antiretroviral prophylaxis to prevent mother-to-child HIV transmission through breastfeeding-the Kisumu Breastfeeding Study, Kenya: a clinical trial. PLoS Med. 2011;8:e1001015. doi:10.1371/journal.pmed.1001015

27. Markos E, Worku A, Davey G. Adherence to ART in PLWHA at Yirgalem Hospital, South Ethiopia. Ethiop J Health Dev. 2008.

28. Bello SI. HIV/AIDS patients' adherence to antiretroviral therapy in Sobi Specialist Hospital, Ilorin, Nigeria. Glob J Med Res. 2011.

29. Igwegbe AO, Ugboaja JO, Nwajiaku LA. Prevalence and determinants of nonadherence to antiretroviral therapy among HIV-positive pregnant women in Nnewi, Nigeria. Int J Med Med Sci. 2010.

30. Ekama SO, Herbertson EC, Addeh EJ, et al. Pattern and determinants of antiretroviral drug adherence among Nigerian pregnant women. J Pregnancy. 2012;2012:851810. doi:10.1155/2012/851810

31. Hagos A, Sarker M Adherence to combined ARV prophylaxis among HIV positive pregnant and lactating women in Addis Ababa health centers, Ethiopia. In: 6th international conference on HIV treatment and prevention adherence; May 22-24, 2011; Miami. Poster number 69033 


\section{Publish your work in this journal}

HIV/AIDS - Research and Palliative Care is an international, peerreviewed open-access journal focusing on advances in research in HIV, its clinical progression and management options including antiviral treatment, palliative care and public healthcare policies to control viral spread. The manuscript management system is completely online and includes a very quick and fair peer-review system, which is all easy to use. Visit http://www.dovepress.com/testimonials.php to read real quotes from published authors.

Submit your manuscript here: https://www.dovepress.com/hivaids—research-and-palliative-care-journal 